\title{
POLYNOMIAL INTEGRABILITY OF THE HAMILTONIAN SYSTEMS WITH HOMOGENEOUS POTENTIAL OF DEGREE -2
}

\author{
JAUME LLIBRE ${ }^{1}$, ADAM MAHDI $^{2}$ AND CLAUDIA VALLS ${ }^{3}$
}

Abstract. We characterize the analytic integrability of Hamiltonian systems
with Hamiltonian $H=\frac{1}{2} \sum_{i=1}^{2} p_{i}^{2}+V\left(q_{1}, q_{2}\right)$, having homogeneous potential
$V\left(q_{1}, q_{2}\right)$ of degree -2 .

\section{INTRODUCTION}

We consider $\mathbb{C}^{4}$ as a symplectic linear space with canonical variables $q=\left(q_{1}, q_{2}\right)$ and $p=\left(p_{1}, p_{2}\right)$. We are interested in Hamiltonian systems defined by the Hamiltonian function

$$
H=\frac{1}{2} \sum_{i=1}^{2} p_{i}^{2}+V(q)
$$

where $V(q)=V\left(q_{1}, q_{2}\right)$ is a homogeneous function of degree $k$. To be more precise we consider the following system of four differential equations

$$
\dot{q}_{i}=p_{i}, \quad \dot{p}_{i}=-\frac{\partial V}{\partial q_{i}}, \quad i=1,2 .
$$

Let $A=A(q, p)$ and $B=B(q, p)$ be two functions. Then their Poisson bracket $\{A, B\}$ is given by

$$
\{A, B\}=\sum_{i=1}^{2}\left(\frac{\partial A}{\partial q_{i}} \frac{\partial B}{\partial p_{i}}-\frac{\partial A}{\partial p_{i}} \frac{\partial B}{\partial q_{i}}\right) .
$$

We say that functions $A$ and $B$ are in involution if $\{A, B\}=0$. We say that a non-constant function $F=F(q, p)$ is a first integral for the Hamiltonian system (2) if it commutes with the Hamiltonian function $H$, i.e. $\{H, F\}=0$. Since the Poisson bracket is antisymmetric it is clear that $H$ itself is always a first integral. We say that a 2-degree of freedom Hamiltonian system (2) is completely or Liouville integrable if it has 2 functionally independent first integrals: $H$, and an additional one $F$, which are in involution. As usual $H$ and $F$ are functionally independent if their gradients are linearly independent at all points of $\mathbb{C}^{4}$ except perhaps in a zero Lebesgue set.

First we recall basic properties of system $(2)$. Let $\mathrm{PO}_{2}(\mathbb{C})$ denote the group of $2 \times 2$ complex matrices $A$ such that $A A^{T}=\alpha I$, where $I$ is the identity matrix and $\alpha \in \mathbb{C} \backslash\{0\}$. We say that potentials $V_{1}(q)$ and $V_{2}(q)$ are equivalent if there exists

2010 Mathematics Subject Classification. 37J35, 34Cxx.

Key words and phrases. natural Hamiltonian systems with 2-degrees of freedom, homogeneous polynomial potential.

Tel. 34935811303 and Fax 34935812790 of the corresponding author, J. Llibre. 


\begin{tabular}{|l||l|}
\hline Case & Potential \\
\hline \hline$V_{1}$ & $q_{1}^{3}$ \\
$V_{2}$ & $q_{1}^{3} / 3+c q_{2}^{3} / 3$ \\
$V_{3}$ & $a q_{1}^{3} / 3+q_{1}^{2} q_{2} / 2+q_{2}^{3} / 6$ \\
$V_{4}$ & $q_{1}^{2} q_{2} / 2+q_{2}^{3}$ \\
$V_{5}$ & $\pm i 7 q_{1}^{3} / 15+q_{1}^{2} q_{2} / 2+q_{2}^{3} / 15$ \\
$V_{6}$ & $q_{1}^{2} q_{2} / 2+8 q_{2}^{3} / 3$ \\
$V_{7}$ & $\pm i 17 \sqrt{14} q_{1}^{3} / 90+q_{1}^{2} q_{2} / 2+q_{2}^{3} / 45$ \\
$V_{8}$ & $\pm i \sqrt{3} q_{1}^{3} / 18+q_{1}^{2} q_{2} / 2+q_{2}^{3}$ \\
$V_{9}$ & $\pm i 3 \sqrt{3} q_{1}^{3} / 10+q_{1}^{2} q_{2} / 2+q_{2}^{3} / 45$ \\
$V_{10}$ & $\pm i 11 \sqrt{3} q_{1}^{3} / 45+q_{1}^{2} q_{2} / 2+q_{2}^{3} / 10$ \\
\hline
\end{tabular}

TABLE 1. All nonequivalent integrable homogeneous potentials of degree 3.

a matrix $A \in \mathrm{PO}_{2}(\mathbb{C})$ such that $V_{1}(q)=V_{2}(A q)$. So we divide all potentials into equivalent classes. Here a potential means a class of equivalent potentials in the above sense. This definition of equivalent potentials is motivated by the following simple lemma. For a proof see [8].

Lemma 1. Let $V_{1}$ and $V_{2}$ be two equivalent potentials. If Hamiltonian system (2) is integrable with potential $V_{1}$ then it is also integrable with $V_{2}$.

In the beginning of 80's all integrable Hamiltonian systems (1) with homogeneous polynomial potential of degree at most 5 and having a second polynomial first integral up to degree 4 in the variables $p_{1}$ and $p_{2}$ were found, see $[14,5,3,6,2]$ and also [7] for the list of corresponding additional first integrals. We remark that all these first integrals are polynomials in the variables $p_{1}, p_{2}, q_{1}$ and $q_{2}$. The main tools used there in order to identify these integrable systems were Painlevé test [4] and direct methods [8].

An elegant result related with the integrability of Hamiltonian systems with a homogeneous polynomial potential was given by Morales and Ramis (see [13, p. 100] and references therein), which gives the necessary condition for the complete meromorphic integrability of such systems. Using the result of Morales-Ramis, Maciejewski and Przybylska [10] gave a necessary and sufficient condition for the complete meromorphic integrability of Hamiltonian systems with the homogeneous polynomial potential of degree 3 . The list of nonequivalent integrable homogeneous potentials of degree 3 is given in Table 1. Later on in [11] the same authors studied, among other things, the meromorphic integrability of the class of Hamiltonian systems with a homogeneous polynomial potential of degree 4 . They proved that except for the family of potentials

$$
V=\frac{1}{2} a q_{1}^{2}\left(q_{1}+i q_{2}\right)^{2}+\frac{1}{4}\left(q_{1}^{2}+q_{2}^{2}\right)^{2}
$$




\begin{tabular}{|l||l|}
\hline Case & \multicolumn{1}{|c|}{ Potential } \\
\hline \hline$V_{i}$ & $\alpha\left(q_{2}-i q_{1}\right)^{i}\left(q_{2}+i q_{1}\right)^{4-i} \quad$ for $i=0,1,2,3,4$. \\
$V_{5}$ & $\alpha q_{2}^{4}$ \\
$V_{6}$ & $\alpha q_{1}^{4} / 4+q_{2}^{4}$ \\
$V_{7}$ & $4 q_{1}^{4}+3 q_{1}^{2} q_{2}^{2}+q_{2}^{4} / 4$ \\
$V_{8}$ & $2 q_{1}^{4}+3 q_{1}^{2} q_{2}^{2} / 2+q_{2}^{4} / 4$ \\
$V_{9}$ & $\left(q_{1}^{2}+q_{2}^{2}\right)^{2} / 4$ \\
$V_{10}$ & $-q_{1}^{2}\left(q_{1}+i q_{2}\right)^{2}+\left(q_{1}^{2}+q_{2}^{2}\right)^{2} / 4$ \\
\hline
\end{tabular}

TABLE 2. Nonequivalent integrable homogeneous potentials of degree 4.

only these systems with potentials $V_{i}$ for $i=0,1, \ldots, 8$ given in Table 2 are the nonequivalent integrable homogeneous potentials of degree 4 . In [9] we proved that for the family (3) only the potentials $V_{9}$ and $V_{10}$ of Table 2 are integrable.

In this paper we classify the analytic integrability of the Hamiltonian systems (2) with homogeneous potentials of degrees $k=2, k=1, k=0, k=-1$ and $k=-2$. So at this moment the analytic integrability of the Hamiltonian systems (2) with homogeneous potentials of degrees $k=-2,-1,0,1,2,3,4$ has been characterized.

\section{Homogeneous potentials of Degrees $2,1,0$ AND -1}

For the sake of completeness we summarize here the trivial results related to the integrability of the Hamiltonian systems with the homogeneous potential of degree $2,1,0$ and -1 being either a polynomial or an inverse of the polynomial. It turns out that all those systems are completely integrable, with a polynomial additional first integral.

Theorem 2. Hamiltonian systems (2) with the homogeneous potential $V$ and one corresponding additional polynomial first integral $I$ :

$$
\begin{array}{ll}
V=a q_{1}^{2}+b q_{1} q_{2}+c q_{2}^{2}, & I=b^{2} q_{1}^{2}+4 b c q_{2} q_{1}+\left(b^{2}+4 c^{2}-4 a c\right) q_{2}^{2}-2(a-c) p_{2}^{2}+2 b p_{1} p_{2}, \\
V=a q_{1}+b q_{2}, & I=a p_{2}-b p_{1}, \\
V=a, & I=p_{1}, \\
V=1 /\left(a q_{1}+b q_{2}\right), & I=a p_{2}-b p_{1}, \\
\text { where } a, b, c \in \mathbb{C} \text { and } V \not \equiv 0 .
\end{array}
$$

Proof. The theorem follows by a straightforward computation.

\section{Homogeneous potential of Degree -2}

In this section we consider Hamiltonian systems (2) with a homogeneous potential of the form

$$
V=V(q)=\frac{1}{a q_{1}^{2}+b q_{1} q_{2}+c q_{2}^{2}} \quad \text { with } a, b, \text { or } c \text { nonzero. }
$$


As we shall see only few of these potentials of degree -2 will be analytically integrable, however all of them are rationally integrable with the additional wellknown first integral

$$
I=\frac{1}{2}\left(q_{1} p_{2}-q_{2} p_{1}\right)^{2}+\left(q_{1}^{2}+q_{2}^{2}\right) V(q),
$$

see for more details [1] and [12].

Our main results are the following two theorems (Theorems 3 and 4).

Theorem 3. The following statements hold.

(a) The polynomial integrability of the Hamiltonian system (2) with homogeneous potential (4) is equivalent to study the polynomial integrability of Hamiltonian system (2) with homogeneous potential $V=1 /\left(a q_{1}^{2}+c q_{2}^{2}\right)$.

(b) The Hamiltonian system (2) with homogeneous potential $V=1 /\left(a q_{1}^{2}+c q_{2}^{2}\right)$ is completely integrable with an additional polynomial first integral if and only if either $c=0$, or $c \neq 0$ and $a \in\{0, c\}$. Moreover this additional first integral is $p_{2}$ if $c=0 ; p_{1}$ if $a=0$ and $q_{1} p_{2}-q_{2} p_{1}$ if $a=c$.

We consider polynomial differential systems of the form

$$
\frac{d x}{d t}=\dot{x}=P(x), \quad x=\left(x_{1}, x_{2}, x_{3}, x_{4}\right) \in \mathbb{C}^{4},
$$

with $P(x)=\left(P_{1}(x), P_{2}(x), P_{3}(x), P_{4}(x)\right)$ and $P_{i} \in \mathbb{C}\left[x_{1}, x_{2}, x_{3}, x_{4}\right]$ for $i=1,2,3,4$. We say that system (5) is weight-homogeneous if there exist $s=\left(s_{1}, s_{2}, s_{3}, s_{4}\right) \in \mathbb{Z}^{4}$ and $d \in \mathbb{Z}$ such that

$$
P_{i}\left(\alpha^{s_{1}} x_{1}, \alpha^{s_{2}} x_{2}, \alpha^{s_{3}} x_{3}, \alpha^{s_{4}} x_{4}\right)=\alpha^{s_{i}-1+d} P_{i}\left(x_{1}, x_{2}, x_{3}, x_{4}\right), \quad i=1,2,3,4,
$$

for arbitrary $\alpha \in \mathbb{R}^{+}=\{\alpha \in \mathbb{R}, \alpha>0\}$. We call $s=\left(s_{1}, s_{2}, s_{3}, s_{4}\right)$ the weight exponent of system (5) and $d$ the weight degree with respect to the weight exponent $s$. We say that a polynomial $F\left(x_{1}, x_{2}, x_{3}, x_{4}\right)$ is a weight-homogeneous polynomial with weight exponent $s$ and weight degree $n$ if

$$
F\left(\alpha^{s_{1}} x_{1}, \alpha^{s_{2}} x_{2}, \alpha^{s_{3}} x_{3}, \alpha^{s_{4}} x_{4}\right)=\alpha^{n} F\left(x_{1}, x_{2}, x_{3}, x_{4}\right) .
$$

We note that Hamiltonian system (2) with homogeneous potential (6) is a weighthomogeneous polynomial differential system with weight exponent $\left(s_{1}, s_{2}, s_{3}, s_{4}\right)=$ $(-1,-1,1,1)$ and weight degree $d=3$. Indeed with those values of $d$ and $s_{i}$, $i=1,2,3,4$ we can easily show

$$
\alpha^{s_{1}-1+d}=\alpha^{s_{3}}, \quad \alpha^{s_{2}-1+d}=\alpha^{s_{4}}, \quad \alpha^{s_{3}-1+d}=\alpha^{-3 s_{1}}, \quad \alpha^{s_{4}-1+d}=\alpha^{-3 s_{2}},
$$

for an arbitrary $\alpha \in \mathbb{R}^{+}$. It is well-known (see for instance Proposition 1 of [9]) that the study of the existence of analytic first integrals of a weight-homogeneous polynomial differential system reduces to the study of the existence of a weighthomogeneous polynomial first integrals. This fact together with Theorem 3 states the following main theorem.

Theorem 4. The Hamiltonian system (2) with homogeneous potential (6) is completely integrable with an additional analytic first integral if and only if either $c=0$, or $c \neq 0$ and $a \in\{0, c\}$.

The following lemma proves statement (a) of Theorem 3. 
Lemma 5. Let $F(q)=a q_{1}^{2}+b q_{1} q_{2}+c q_{2}^{2}$. Then there exists a change of variables $q=A \bar{q}$, where $A \in \mathrm{PO}_{2}(\mathbb{C})$ such that

$$
F(A \bar{q})=\alpha \bar{q}_{1}^{2}+\beta \bar{q}_{2}^{2} .
$$

Proof. We can assume that $b \neq 0$, otherwise there is nothing to prove. Let

$$
\left(\begin{array}{l}
q_{1} \\
q_{2}
\end{array}\right)=\left(\begin{array}{cc}
a_{1} & a_{2} \\
-a_{2} & a_{1}
\end{array}\right)\left(\begin{array}{c}
\bar{q}_{1} \\
\bar{q}_{2}
\end{array}\right) \text {. }
$$

Then

$$
F(A \bar{q})=\bar{\alpha} \bar{q}_{1}^{2}+\bar{\beta} \bar{q}_{1} \bar{q}_{2}+\bar{\gamma} \bar{q}_{2}^{2}
$$

with

$$
\begin{aligned}
\bar{\alpha} & =\left(a a_{1}^{2}-a_{1} a_{2} b+a_{2}^{2} c\right) \\
\bar{\beta} & =2 a a_{1} a_{2}+a_{1}^{2} b-a_{2}^{2} b-2 a_{1} a_{2} c \\
\bar{\gamma} & =\left(a a_{2}^{2}+a_{1} a_{2} b+a_{1}^{2} c\right) .
\end{aligned}
$$

Taking

$$
a_{1}=\frac{a_{2}(c-a)+\sqrt{a_{2}^{2}\left(b^{2}+(a-c)^{2}\right)}}{b}
$$

we get $\bar{\beta}=0$. form

The above lemma implies that we can work with a homogeneous potential of the

$$
V=\frac{1}{a q_{1}^{2}+c q_{2}^{2}}, \quad \text { with } a \text { or } c \text { nonzero. }
$$

First we consider the case $a c(c-a) \neq 0$. We recall that we have the Hamiltonian system

$$
\dot{q}_{1}=p_{1}, \quad \dot{q}_{2}=p_{2}, \quad \dot{p}_{1}=\frac{2 a q_{1}}{\left(a q_{1}^{2}+c q_{2}^{2}\right)^{2}}, \quad \dot{p}_{2}=\frac{2 c q_{2}}{\left(a q_{1}^{2}+c q_{2}^{2}\right)^{2}},
$$

where the dot in (7) denotes the derivative with respect to $t$. Now we take the new independent variable $\tau$ defined by $d t=\left(a q_{1}^{2}+c q_{2}^{2}\right)^{2} d \tau$. Then system (7) becomes

$$
\dot{q}_{1}=p_{1}\left(a q_{1}^{2}+c q_{2}^{2}\right)^{2}, \dot{q}_{2}=p_{2}\left(a q_{1}^{2}+c q_{2}^{2}\right)^{2}, \dot{p}_{1}=2 a q_{1}, \dot{p}_{2}=2 c q_{2},
$$

where now the dot denotes the derivative with respect to $\tau$. Changing the variables $\left(q_{1}, q_{2}, p_{1}, p_{2}\right) \rightarrow\left(q_{1}, q_{2}, p_{1}, T\right)$, where $T=q_{2} p_{1}-q_{1} p_{2}$, system (8) writes

$$
\begin{aligned}
\dot{q}_{1} & =p_{1}\left(a q_{1}^{2}+c q_{2}^{2}\right)^{2}, \\
\dot{q}_{2} & =\frac{q_{2} p_{1}-T}{q_{1}}\left(a q_{1}^{2}+c q_{2}^{2}\right)^{2}, \\
\dot{p}_{1} & =2 a q_{1}, \\
\dot{T} & =2(a-c) q_{1} q_{2} .
\end{aligned}
$$

With this change of variables we put in evidence the first integral when $a=c$.

If we denote by $F\left(q_{1}, q_{2}, p_{1}, p_{2}\right) \in \mathbb{C}\left[q_{1}, q_{2}, p_{1}, p_{2}\right]$ a polynomial first integral of (8), then in the variables $\left(q_{1}, q_{2}, p_{1}, T\right)$ it writes

$$
F\left(q_{1}, q_{2}, p_{1}, T\right)=\sum_{j=-n}^{n} f_{j}\left(q_{2}, p_{1}, T\right) q_{1}^{j},
$$


where $f_{j}\left(q_{2}, p_{1}, T\right) \in \mathbb{C}\left[q_{2}, p_{1}, T\right]$. By definition $F$ is a first integral of (9) if and only if $F$ is non-constant and

$$
\left(a q_{1}^{2}+c q_{2}^{2}\right)^{2}\left(\frac{\partial F}{\partial q_{1}} p_{1}+\frac{\partial F}{\partial q_{2}} \frac{q_{2} p_{1}-T}{q_{1}}\right)+2 q_{1}\left(a \frac{\partial F}{\partial p_{1}}+\frac{\partial F}{\partial T}(a-c) q_{2}\right)=0 .
$$

We define the following differential operators that act on $f_{j}=f_{j}\left(q_{2}, p_{1}, T\right) \in$ $\mathbb{R}\left[q_{2}, p_{1}, T\right]$ :

$$
\begin{aligned}
& \mathcal{A}\left[f_{j}\right]:=j p_{1} f_{j}+\left(q_{2} p_{1}-T\right) \frac{\partial f_{j}}{\partial q_{2}}, \\
& \mathcal{B}\left[f_{j}\right]:=c q_{2}^{2} a\left(q_{2} p_{1}-T\right) \frac{\partial f_{j}}{\partial q_{2}}+a \frac{\partial f_{j}}{\partial p_{1}}+(a-c) q_{2} \frac{\partial f_{j}}{\partial T}+j a c q_{2}^{2} p_{1} f_{j} .
\end{aligned}
$$

Computing the different coefficients of $q_{1}^{j}$ in (11) for $j=-n-1, \ldots, n+3$ we get that $F$ is a first integral of (9) if and only if

$$
\begin{aligned}
& c^{2} q_{2}^{4} \mathcal{A}\left[f_{i}\right]=0, \quad \text { for } i=-n,-n+1, \\
& 2 \mathcal{B}\left[f_{i}\right]+c^{2} q_{2}^{4} \mathcal{A}\left[f_{i+2}\right]=0, \quad \text { for } i=-n,-n+1, \\
& c^{2} q_{2}^{4} \mathcal{A}\left[f_{i}\right]+2 \mathcal{B}\left[f_{i-2}\right]+a^{2} \mathcal{A}\left[f_{i-4}\right]=0, \quad \text { for } i=-n+4, \ldots, n, \\
& 2 \mathcal{B}\left[f_{i}\right]+a^{2} \mathcal{A}\left[f_{i-2}\right]=0, \quad \text { for } i=n-1, n, \\
& a^{2} \mathcal{A}\left[f_{i}\right]=0, \text { for } i=n-1, n \text {. }
\end{aligned}
$$

We shall prove that if $a c(a-c) \neq 0$, then $F=$ const and consequently it is not a first integral. The proof will follow from the following two lemmas.

Lemma 6. Let $F$ be as in (10) and $a c(a-c) \neq 0$. If $F$ is first integral of (9), then $f_{j}\left(q_{2}, p_{1}, T\right)=0$ for $j=1, \ldots, n$.

Proof. From (12) we consider $a^{2} \mathcal{A}\left[f_{n}\right]=0$. Using that $a \neq 0$, the solution is $f_{n}=\alpha /\left(T-q_{2} p_{1}\right)^{n}$, where $\alpha=\alpha\left(p_{1}, T\right)$. Since $f_{n} \in \mathbb{C}\left[q_{2}, p_{1}, T\right]$ we conclude that $f_{n}=0$. Similarly, from $a^{2} \mathcal{A}\left[f_{n-1}\right]$ we show that $f_{n-1}=0$. Now using that $\mathcal{B}[0]=0$, and $f_{n}=f_{n-1}=0$ the conditions

$$
2 \mathcal{B}\left[f_{i}\right]+a^{2} \mathcal{A}\left[f_{i-2}\right]=0 \quad \text { for } \quad i=n-1, n,
$$

implies that $\mathcal{A}\left[f_{n-2}\right]=\mathcal{A}\left[f_{n-3}\right]=0$. Thus using the arguments for solving $a^{2} \mathcal{A}\left[f_{n}\right]=0$ we obtain that as long as $n-3 \geq 1$ we get $f_{n-2}=f_{n-3}=0$. If $n=4$ we are done. If $n \geq 5$, then we proceed by induction. Assume that $f_{n}=f_{n-1}=\ldots=f_{j+1}=0$, where $j \geq 1$. We shall show that $f_{j}=0$. Now we consider condition (12) for $i=j+4$, that is,

$$
c^{2} q_{2}^{4} \mathcal{A}\left[f_{j+4}\right]+2 \mathcal{B}\left[f_{j+2}\right]+a^{2} \mathcal{A}\left[f_{j}\right]=0 .
$$

Since $f_{j+4}=f_{j+2}=0$, we have $\mathcal{A}\left[f_{j+4}\right]=\mathcal{B}\left[f_{j+2}\right]=0$. Thus condition (13) reduces to $\mathcal{A}\left[f_{j}\right]=0$. Since $j \geq 1$, the only polynomial solution of this differential equation is $f_{j}=0$.

Lemma 7. Let $F$ be as in (10) and $a c(a-c) \neq 0$. If $F$ is first integral of (9), then $f_{j}\left(q_{2}, p_{1}, T\right)=0$ for $j=-n,-n+1 \ldots,-1$ and $f_{0}\left(q_{2}, p_{1}, T\right)=$ constant.

Proof. Consider (12) for $i=-n,-n+1$, that is, $c^{2} q_{2}^{4} \mathcal{A}\left[f_{-n}\right]=0$ and $c^{2} q_{2}^{4} \mathcal{A}\left[f_{-n+1}\right]=$ 0 . Since $c \neq 0$ this implies that $\mathcal{A}\left[f_{-n}\right]=\mathcal{A}\left[f_{-n+1}\right]=0$ and solving it we get

$$
f_{-n}=\left(q_{2} p_{1}-T\right)^{n} \alpha_{-n}, \quad \text { and } \quad f_{-n+1}=\left(q_{2} p_{1}-T\right)^{n-1} \alpha_{-n+1},
$$


where $\alpha_{-n}=\alpha_{-n}\left(p_{1}, T\right)$ and $\alpha_{-n+1}=\alpha_{-n+1}\left(p_{1}, T\right)$ are polynomials. Now we consider the condition $2 \mathcal{B}\left[f_{i}\right]+c^{2} q_{2}^{4} \mathcal{A}\left[f_{i+2}\right]=0$, for $i=-n,-n+1$ and we shall use (14). Thus for $i=-n$ we get

$$
f_{-n+2}=\left(q_{2} p_{1}-T\right)^{n-2}\left[\alpha_{-n+2}+\beta_{-n+2}\right],
$$

where $\alpha_{-n+2}=\alpha_{-n+2}\left(p_{1}, T\right)$ is an integral constant and

$\beta_{-n+2}=\frac{1}{3 c^{2} q_{2}^{3}}\left(3 n c q_{2} \alpha_{-n}-3(a-c) q_{2}\left(T-2 q_{2} p_{1}\right) \frac{\partial \alpha_{-n}}{\partial T}+a\left(3 q_{2} p_{1}-2 T\right) \frac{\partial \alpha_{-n}}{\partial p_{1}}\right)$.

Since $f_{-n+2}$ is a polynomial, $\beta_{-n+2}$ also is a polynomial. In the expression of $\beta_{-n+2}$ there are terms $(a-c) p_{1} q_{2}^{-1} \frac{\partial \alpha_{-n}}{\partial T}$ and $-\frac{2}{3} a T q_{2}^{-3} \frac{\partial \alpha_{-n}}{\partial p_{1}}$. Since $f_{n-2}$ is a polynomial we obtain that

$$
\frac{\partial \alpha_{-n}}{\partial T}=\frac{\partial \alpha_{-n}}{\partial p_{1}}=0
$$

So $\alpha_{-n}=$ constant. Moreover in $\beta_{-n+2}$ we also have the term $n c^{-1} q_{2}^{-2} \alpha_{-n}$. Again since $f_{-n+2}$ is a polynomial $\alpha_{-n}=0$, thus $f_{-n}=0$. Working with $f_{-n+1}$ similarly as with $f_{-n}$ we obtain

$$
f_{-n+3}=\left(q_{2} p_{1}-T\right)^{n-3}\left[\alpha_{-n+3}+\beta_{-n+3}\right],
$$

where $\alpha_{-n+3}=\alpha_{-n+3}\left(p_{1}, T\right)$ and

$\beta_{-n+3}=\frac{1}{3 c^{2} q_{2}^{3}}\left(3(n-1) c q_{2} \alpha_{-n+1}-3(a-c) q_{2}\left(T-2 q_{2} p_{1}\right) \frac{\partial \alpha_{-n+1}}{\partial T}+a\left(3 q_{2} p_{1}-2 T\right) \frac{\partial \alpha_{-n+1}}{\partial p_{1}}\right)$.

Similarly as in the previous case we conclude that $\alpha_{-n+1}=0$. In summary we have proved that $f_{-n}=f_{-n+1}=0$. Now we shall proceed by induction. Assume that $f_{-n}=\ldots=f_{-j-1}=0$ and for $-j \leq-3$. We shall prove that $f_{-j}=0$. Consider (12) for $i=-j$, that is,

$$
c^{2} q_{2}^{4} \mathcal{A}\left[f_{-j}\right]+2 \mathcal{B}\left[f_{-j-2}\right]+a^{2} \mathcal{A}\left[f_{-j-4}\right]=0 .
$$

Since by induction hypothesis $f_{-j-4}=f_{-j-2}=0$, we have $\mathcal{A}\left[f_{-j-4}\right]=\mathcal{B}\left[f_{-j-2}\right]=$ 0 . Thus, condition (15) reduces to $\mathcal{A}\left[f_{-j}\right]=0$. This implies that $f_{-j}=\left(q_{2} p_{1}-\right.$ $T)^{j} \alpha_{-j}$, where again $\alpha_{-j}=\alpha_{-j}\left(p_{1}, T\right)$. Now considering (12) for $i=-j+2$ we get

$$
c^{2} q_{2}^{4} \mathcal{A}\left[f_{-j+2}\right]+2 \mathcal{B}\left[f_{-j}\right]+a^{2} \mathcal{A}\left[f_{-j-2}\right]=0 .
$$

Taking into account that that $\mathcal{A}\left[f_{-j-2}\right]=0$ as well as $f_{-j}=\left(q_{2} p_{1}-T\right)^{j} \alpha_{-j}$ the solution of (16) writes

$$
f_{-j+2}=\left(q_{2} p_{1}-T\right)^{j-2}\left[\alpha_{-j+2}+\beta_{-j+2}\right],
$$

where

$$
\beta_{-j+2}=\frac{1}{3 c^{2} q_{2}^{3}}\left(3 j c q_{2} \alpha_{-j}-3(a-c) q_{2}\left(T-2 q_{2} p_{1}\right) \frac{\partial \alpha_{-j}}{\partial T}+a\left(3 q_{2} p_{1}-2 T\right) \frac{\partial \alpha_{-j}}{\partial p_{1}}\right) .
$$

Again, since $\beta_{-j+2}$ has to be a polynomial the same argument as before allows to deduce that $\alpha_{-j}=0$, therefore $f_{-j}=0$.

Following the induction steps we have proved that $f_{-n}=\ldots=f_{-3}=0, f_{-2}=$ $\left(T-q_{2} p_{1}\right)^{2} \alpha_{-2}$ and $f_{-1}=\left(T-q_{2} p_{1}\right) \alpha_{-1}$, where $\alpha_{-1}=\alpha_{-1}\left(p_{1}, T\right)$ and $\alpha_{-2}=$ $\alpha_{-2}\left(p_{1}, T\right)$. Consider again (12) for $i=1$, that is,

$$
c^{2} q_{2}^{4} \mathcal{A}\left[f_{1}\right]+2 \mathcal{B}\left[f_{-1}\right]+a^{2} \mathcal{A}\left[f_{-3}\right]=0 .
$$


By Lemma $6 f_{1}=0$ so this implies $\mathcal{A}\left[f_{1}\right]=0$. Since by the induction process $f_{-3}=0$, and $f_{-1}=\left(T-q_{2} p_{1}\right) \alpha_{-1}$ the solution of $(17)$ is given by

$$
\alpha_{-1}=\gamma\left(T+\frac{c-a}{a} q_{2} p_{1}\right) /\left(a\left(T-q_{2} p_{1}\right)\right), \quad \text { where } \quad \gamma \in \mathbb{C} .
$$

Again since $\alpha_{-1}=\alpha_{-1}\left(p_{1}, T\right)$ is a polynomial and $(c-a) / a \neq 0$ we conclude that $\gamma=0$, thus $\alpha_{-1}=0$ which implies that $f_{-1}=0$. To show that $f_{-2}=0$ and that $f_{0}=f_{0}\left(p_{1}, T\right)$, that is, $f_{0}$ does not depend on $q_{2}$ consider (12) for $i=0$, that is,

$$
c^{2} q_{2}^{4} \mathcal{A}\left[f_{0}\right]+2 \mathcal{B}\left[f_{-2}\right]+a^{2} \mathcal{A}\left[f_{-4}\right]=0 .
$$

Since $\mathcal{A}\left[f_{-4}\right]=0, f_{-2}=\left(T-q_{2} p_{1}\right)^{2} \alpha_{-2}$ and $\alpha_{-2}=\alpha_{-2}\left(p_{1}, T\right)$ solving (18) we get

$$
f_{0}=\alpha_{0}+\beta_{0},
$$

where $\alpha_{0}=\alpha_{0}\left(p_{1}, T\right)$ and

$$
\beta_{0}=\frac{1}{3 c q_{2}^{3}}\left(6 c q_{2} \alpha_{-2}-3(a-c) q_{2}\left(T-2 q_{2} p_{1}\right) \frac{\partial \alpha_{-2}}{\partial T}+a\left(3 q_{2} p_{1}-2 T\right) \frac{\partial \alpha_{-2}}{\partial p_{1}}\right) .
$$

Since $\beta_{0}$ has to be a polynomial, using the same arguments for proving that $\beta_{-n+2}=$ 0 we conclude that $\alpha_{-2}=0$, and consequently $\beta_{0}=0$. Thus $f_{-2}=0$, and therefore $f_{0}=\alpha_{0}\left(p_{1}, T\right)$.

Finally we consider (12) for $i=2$. Since by Lemma 6 we have that $f_{2}=0$, as well as $f_{0}=f_{0}\left(p_{1}, T\right)$ we get

$$
a \frac{\partial f_{0}}{\partial p_{1}}+(a-c) q_{2} \frac{\partial f_{0}}{\partial T}=0
$$

Its solution is of the form

$$
f_{0}\left(p_{1}, T\right)=F\left(T+\frac{c-a}{a} q_{2} p_{1}\right) .
$$

Since $a(a-c) \neq 0$ and $f_{0}$ does not depend on $q_{2}$ we get that $f_{0}=$ constant which ends the proof.

Proof of Theorem 3. If $c=0$ then $p_{2}$ is an additional polynomial first integral and the corresponding Hamiltonian system (2) with potential (6) is completely integrable. So we can assume that $c \neq 0$.

There are at least two values of $a$ for which system (7) is completely integrable. These cases are $a=0$ with additional first integral $p_{1}$ and $a=c$ with additional first integral $q_{1} p_{2}-q_{2} p_{1}$. We note that in both cases the additional first integral is a polynomial. The rest of the proof follows directly from Lemmas 6 and 7 .

\section{ACKnowledgements}

The first author is supported by the grants MCYT/FEDER MTM 2008-03437 and Generalitat de Catalunya 2009SGR410 and by ICREA Academia. The third author is partially supported by FCT through CAMGDS, Lisbon. 


\section{REFERENCES}

[1] A.V. Borisov, A.A. Kilin, and I.S. Mamaev, Multiparticle Systems. The Algebra of Integrals and Integrable Cases, Regular and Chaotic Dynamics, 14 (2009), 18-41.

[2] T. Bountis, H. Segur, and F. Vivaldi, Integrable Hamiltonian systems and the Painlevé property, Phys. Rev. A (3) 25 (1982), no. 3, 1257-1264.

[3] Y.F. Chang, M. Tabor, and J. Weiss, Analytic structure of the Hénon-Heiles Hamiltonian in integrable and nonintegrable regimes, J. Math. Phys. 23 (1982), no. 4, 531-538.

[4] A. Goriely, Integrability and nonintegrability of dynamical systems, Advanced Series in Nonlinear Dynamics, vol. 19, World Scientific Publishing Co. Inc., River Edge, NJ, 2001.

[5] B. Grammaticos, B. Dorizzi, and R. Padjen, Painlevé property and integrals of motion for the Hénon-Heiles system, Phys. Lett. A 89 (1982), no. 3, 111-113.

[6] L.S. Hall, A theory of exact and approximate configurational invariants, Phys. D 8 (1983), no. 1-2, 90-116.

[7] J. Hietarinta, A search for integrable two-dimensional Hamiltonian systems with polynomial potential, Phys. Lett. A 96 (1983), 273-278.

[8] Direct methods for the search of the second invariant, Phys. Rep. 147 (1987), 87-154.

[9] J. Llibre, A. Mahdi and C. Valls, Analytic integrability of Hamiltonian systems with homogeneous polynomial potential of degree 4, J. Math. Phys. 52, (2011), 012702, 9 pp .

[10] A.J. Maciejewski and M. Przybylska, All meromorphically integrable 2D Hamiltonian systems with homogeneous potential of degree 3, Phys. Lett. A 327 (2004), no. 5-6, 461-473.

[11] _ Darboux points and integrability of Hamiltonian systems with homogeneous polynomial potential, J. Math. Phys. 46 (2005), no. 6, 062901.

[12] _ Necessary conditions for classical super-integrability of a certain family of potentials in constant curvature spaces, J. Phys. A 43 (2010), 382001, 15 pp.

[13] J.J. Morales Ruiz, Differential Galois theory and non-integrability of Hamiltonian systems, Progress in Mathematics, vol. 179, Birkhäuser Verlag, Basel, 1999.

[14] A. Ramani, B. Dorizzi, and B. Grammaticos, Painlevé conjecture revisited, Phys. Rev. Lett. 49 (1982), no. 21, 1539-1541.

1 Departament de Matemàtiques, Universitat Autònoma de Barcelona, 08193 Bellaterra, Barcelona, Catalonia, Spain

E-mail address: jllibre@mat.uab.cat, mahdi@mat.uab.cat

2 Mathematics Department, University of North Carolina at Charlotte, Charlotte, North Carolina 28223, USA

E-mail address: adam.mahdi@uncc.edu

2 Faculty of Applied Mathematics, AGH University of Science and Technology, al. Mickiewicza 30, 30-059 Kraków, Poland

3 Departamento de Matemática, Instituto Superior Técnico, Av. Rovisco Pais 1049001, Lisboa, Portugal

E-mail address: cvalls@math.ist.utl.pt 